\title{
Successful Outcome of Refractory Chronic Constipation by Surgical Treatment: A Series of 34 Patients
}

\author{
Ashok Kumar, ${ }^{1 *}$ HM Lokesh ${ }^{1}$ and Uday C Ghoshal ${ }^{2}$ \\ Departments of ${ }^{1}$ Surgical Gastroenterology and ${ }^{2}$ Gastroenterology, Sanjay Gandhi Post Graduate Institute of Medical Sciences, Lucknow, UP, \\ India
}

\begin{abstract}
Background/Aims
Chronic constipation is commonly managed medically, and surgical options have been advocated in patients with refractory symptoms. We aimed to study the role of surgical procedures in patients with constipation, refractory to medical therapy.

\section{Methods}

Data on 34 surgically managed patients with refractory chronic constipation during a 6-year period (March 2003 to May 2009) were retrospectively analyzed.

Results

All the 34 patients (24 males and 10 females, median age of 45 years [range, 18-77 years]) had symptoms for a long period (median 96 months [range, 12-360 months]) without response to medical treatment including biofeedback. Preopertive investigations included barium enema, colonoscopy, colonic transit study, defecography and anorectal manometry as indicated. Eight patients (23.5\%) had slow transit constipation, $4(11.8 \%)$ had Hirschsprung's disease and $22(64.7 \%)$ had rectal prolapse. Total colectomy and ileo-rectal anastomosis, anterior resection, Delorme's procedure, resection rectopexy and Duhamel's operation were the surgical procedures performed. Though 7 (20.6\%) patients had post operative complications, there was no mortality. One patient whose symptoms recurred following anterior resection was successfully treated by total colectomy and ileo-rectal anastomosis. Median spontaneous bowel movements increased following surgical treatment compared to that while on medical treatment (1 per week [range, 0 to 3 per week] vs. 14 per week [range, 7-28 per week], $P<0.00001$ ). Patients remained well during 3-60 months follow-up $(n=27)$.
\end{abstract}

\section{Conclusions}

Spontaneous bowel movements significantly increased following surgical operation for refractory chronic constipation, nature of which is dependent on underlying etiology and the expertise available. Careful preoperative work-up and selection of patients are critical for obtaining good functional results.

(J Neurogastroenterol Motil 2013;19:78-84)

\section{Key Words}

Constipation; Outcome assessment; Surgery

Received: August 22, 2012 Revised: November 8, 2012 Accepted: November 15, 2012

(c) This is an Open Access article distributed under the terms of the Creative Commons Attribution Non-Commercial License (http://creativecommons org/licenses/by-nc/3.0) which permits unrestricted non-commercial use, distribution, and reproduction in any medium, provided the original work is properly cited.

*Correspondence: Ashok Kumar, MS, FACS, FRCS

Department of Surgical Gastroenterology, Sanjay Gandhi Post Graduate Institute of Medical Sciences, Rae Bareli Road, Lucknow 226014, UP, India

Tel: +91-522-2668700 (ext. 4423, 4401) or +91-522-2998550, Fax: +91-522-2668017 (or 2668078), E-mail: doc.ashokgupta@gmail. com

Financial support: None.

Conflicts of interest: None.

Author contributions: Ashok Kumar: conception and design of the article. HM Lokesh: drafting of the article. Uday C Ghoshal: analysis and interpretation of the data. 


\section{Introduction}

Chronic constipation (CC) is a common complaint at all ages and is commonly associated with symptoms interfering with patient's quality of life. Constipation can result from several factors, like person's life style such as low fiber diet, inadequate fluid intake, consumption of intake of some drugs, slow colonic motility, fecal evacuation disorders and combination of above. ${ }^{1}$ Organic and anatomical causes affect the management strategy. Correction of life style factors and supplementation with laxatives relieve constipation in majority of patients. Surgery will be required in minority of patients who fail medical management. Colectomy for constipation was first described by Sir Arbuthnot Lane a century ago, ${ }^{2}$ and since then various combinations of surgical procedures have been used. Studies regarding surgical management of refractory constipation have been conducted on Western population. No study has addressed the need of surgery and its outcome in Indian population, where the dietary and bowel habits are different, and the indication for surgery may also be different. This study was conducted to assess the postoperative and long-term outcome of surgical management in CC patients, who were refractory to medical management.

\section{Materials and Methods}

Retrospective analysis of prospectively maintained data of 34 surgically managed patients with refractory chronic constipation was performed at Department of Surgical Gastroenterology in Sanjay Gandhi Postgraduate Institute of Medical Sciences (SGPGIMS), Lucknow, a tertiary referral center in northern India from March 2003 to May 2009. Patients were divided into 3 groups according to the indication for surgery, such as slow transit constipation (STC) $(n=8,23.5 \%)$, congenital anomaly like Hirschsprung's disease (HD) $(n=4,11.8 \%)$ and constipation with rectal prolapse $(n=22,64.7 \%)$.

\section{Evaluation and Medical Management}

All patient initially presented to the Department of Gastroenterology at our center, where each of them was evaluated clinically with detailed history and physical examination. CC in these patients was defined by Rome II diagnostic criteria ${ }^{3} ; 2$ or more of the following criteria were present for at least 12 weeks in the preceding 12 months, (1) straining in more than $25 \%$ of defecations, (2) lumpy or hard stools in more than $25 \%$ of defecations, (3) sensation of incomplete evacuation in more than $25 \%$ of defecations, (4) manual maneuvers (e.g., digital evacuation and support of the pelvic floor) to facilitate more than $25 \%$ of defecations and (5) 2 or less bowel movements per week. Details regarding history, spontaneous bowel movements (SBM) per week, clinical examination, and treatment received at other center before referral and associated medical conditions were documented. They were then investigated based on standard protocol using barium enema, colonoscopy, colonic transit time (CTT) study, defecography and anorectal manometry. The investigations were tailored as per the clinical diagnosis; therefore, all patients did not undergo every test. Systemic causes for constipation were ruled out by testing serum thyroxin, thyroid stimulating hormones and postprandial blood sugar levels in all patients. HD patients were referred for surgical management once the diagnosis was made. Remaining patients were referred for surgery after failed medical management using laxatives, enemas and biofeedback therapy. Pressure Biofeedback therapy was performed using a water perfusion manometry system and 2 port low compliance polyvinyl catheter (one port in rectum and another in anal canal) (RedTech, Calabasas, CA, USA). Each session lasted 20 to 30 minutes, in which patient received visual and auditory feedback showing whether he/she could reduce anal sphincter pressure below the target level and rectal pressure above the target pressure. Patient received 2 sessions each day for a period of 14 days. Biofeedback was given only to consenting patients with fecal evacuation disorder (except HD) with or without STC.

CTT study was done using locally manufactured radio-opaque markers in gelatin capsules (SG mark); subjects were asked to ingest 4 capsules at a time ( 5 markers in each capsule) at 0,12 and 24 hours. Subsequently, abdominal X-rays were taken at 36 and 60 hours. STC was diagnosed when more than 30 markers in 36 hours X-ray or more than 14 markers in 60 hours X-ray were retained in colon. ${ }^{4}$

\section{Definitions}

SBM was defined as passage of stools without the use of laxatives or enemas or digital evacuation. Surgery was considered when one or more of the following criteria were present.

(1) Requiring excessive cathartics (failure of osmotic laxatives to work requiring stimulant laxatives or enema on regular basis)

(2) Not responding to cathartics, (less than 3 bowel movements per week and with unusual prolonged straining in spite of high dose of laxatives) 
(3) Fecal impaction and rectal prolapse on rectal examination

(4) Gastrointestinal transit test showing slow colonic transit on CTT

(5) Rectocele confirmed on defecography

(6) Absence of ganglion cells in submucosal and myenteric plexus on rectal biopsy suggesting HD

Gastrointestinal organic diseases were excluded by colonoscopy or barium enema as indicated. Response to treatment was defined by number of SBM per day and it was considered satisfactory with minimum of one SBM per day.

Table 1. Patients Characteristics $(\mathrm{N}=34)$

\begin{tabular}{lc}
\hline Sex $(\mathrm{n}[\%])$ & \\
Male & $24(70.5)$ \\
Female & $10(29.4)$ \\
Age (yr) & \\
Median & 45 \\
Range & $18-77$ \\
Duration of symptoms (yr) & \\
Median & 8 \\
Range & $1-30$ \\
Group wise distribution (n [\%]) & \\
Slow transit constipation & $8(23.5)$ \\
Hirschsprung's disease & $4(11.8)$ \\
Constipation with prolapse & $22(64.7)$ \\
\hline
\end{tabular}

\section{Surgical Management and Follow-up}

Consent for surgery was obtained from all patients after informing the nature of disease, its outcome, treatment options, possible outcomes of surgical management, complication and long-term functional outcomes. Resectional surgical procedures were performed according to the indications for surgery. Close monitoring of patients was done in post-operative period for any complication. Patients were then followed up in outpatient clinic; details regarding SBM per day, need of laxatives and satisfaction with surgical outcome were documented.

\section{Statistical Methods}

Data was entered in SPSS 15.0 statistical package software (SPSS Inc., Chicago, IL, USA). All data are expressed as me-

Table 2. Duration of Symptoms and Medical Therapy (Months)

\begin{tabular}{lcc}
\hline Group & $\begin{array}{c}\text { Duration of } \\
\text { symptoms } \\
\text { (mean [range]) }\end{array}$ & $\begin{array}{c}\text { Duration of } \\
\text { medical therapy } \\
\text { (mean [range]) }\end{array}$ \\
\hline STC $(\mathrm{n}=8)$ & $114(24-300)$ & $100(24-240)$ \\
$\mathrm{HD}(\mathrm{n}=4)$ & $117(12-360)$ & $58(6-120)$ \\
Constipation with & $121(24-360)$ & $104(6-360)$ \\
prolapse $(\mathrm{n}=22)$ & & \\
\hline
\end{tabular}

STC, slow transit constipation; HD, Hirschsprung's disease.
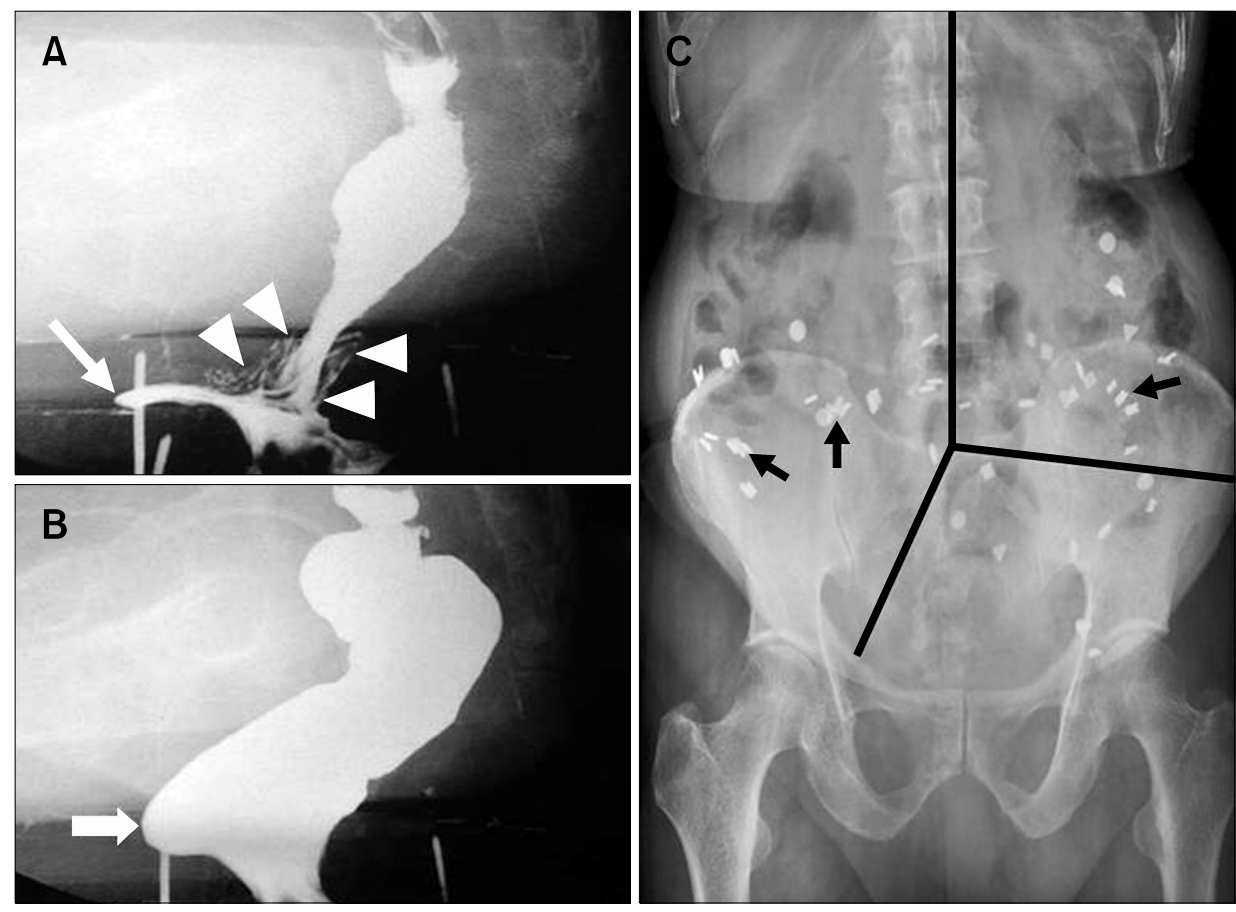

Figure 1. (A) Defecography - showing anterior rectocele (white long arrow) with intra-anal intussusception (white arrow heads) and incomplete rectal evacuation during defecation, (B) Defecography showing rectocele (white short arrow) on straining, (C) Colonic transit study - 60 hourrs abdominal X-ray showing retained markers (black arrows) dispersed throughout the colon. 
dian and range. Statistical comparison of pre-operative and post-operative SBM was done with Wilcoxon signed-rank test and $P$-value of less than 0.05 was considered statistically significant.

\section{Results}

During the study period from March 2003 to May 2009, 34 patients with refractory $\mathrm{CC}$ were surgically treated at our center. There were 24 males $(70.6 \%)$ and 10 females $(29.4 \%)$ in the age range of 18-77 years (median, 45 years). Patient's characteristics are shown in Table 1. All patients had complaints of constipation and feeling of incomplete bowel evacuation for a significantly long period of time. Median duration of symptoms was 96 months (range, 12-360 months) and the median duration of medical management was 60 months (range, 6-360 months). The group wise distribution of duration of symptoms and medical treatment was almost similar except in HD patients; they were referred sooner for surgical intervention once the diagnosis was made (Table 2). HD patients were treated for significantly long period at outside hospital before referral to our center.
All HD patients were found to have dilated colon and proximal rectum on barium enema examination. Four patients with STC showed normal small bowel transit on barium meal follow through examination. Defecography examination revealed internal intussusception in 1 (Fig. 1A), anterior rectocele in 3 patients (Fig. 1B), posterior rectocele in 1, posterior wall ulcer in 1 and rectocele with abnormal pelvic floor descent in 2 patients (Table 3).

On CTT study all eight patients with STC revealed delayed passage of markers. Retention of more than 14 markers dispersed in colon on 60 hours X-ray was seen in 5 patients (Fig. 1C) and retention of more than 14 markers in sigmoid colon and rectum was seen in remaining 3 patients. Two patients of HD who underwent CTT revealed delayed excretion with retention of markers in sigmoid colon and rectum. Anorectal manometry showed absent recto-anal inhibitory reflex, abnormal balloon expulsion ( $>200 \mathrm{~g}$ ) and normal sphincter pressures in all HD patients. Sphincter length was $3.5 \pm 1.5 \mathrm{~cm}$ in HD patients.

Unsuccessful surgical interventions were already performed in 8 patients elsewhere ( 2 in HD group and 6 in prolapse with

Table 3. Investigations Performed

\begin{tabular}{|c|c|c|c|c|c|c|c|c|c|}
\hline & \multicolumn{2}{|c|}{ Barium enema $(\mathrm{n}=7)$} & Colonoscopy $(\mathrm{n}=12)$ & \multicolumn{2}{|c|}{$\operatorname{CTT}(\mathrm{n}=12)$} & \multicolumn{2}{|c|}{$\operatorname{ARM}(\mathrm{n}=21)$} & \multicolumn{2}{|c|}{ Defecogram $(n=8)$} \\
\hline & Normal & Abnormal & Normal Abnormal & Normal & Abnormal & Normal & Abnormal & Normal & Abnormal \\
\hline STC & & 3 & 5 & & 8 & 4 & 3 & & 6 \\
\hline HD & & 4 & 2 & & 2 & & 4 & & \\
\hline Constipation with prolapse & & & 5 & & 2 & 6 & 4 & & 2 \\
\hline
\end{tabular}

CTT, colonic transit study; ARM, anorectal manometry; STC, slow transit constipation; HD, Hirschsprung's disease.

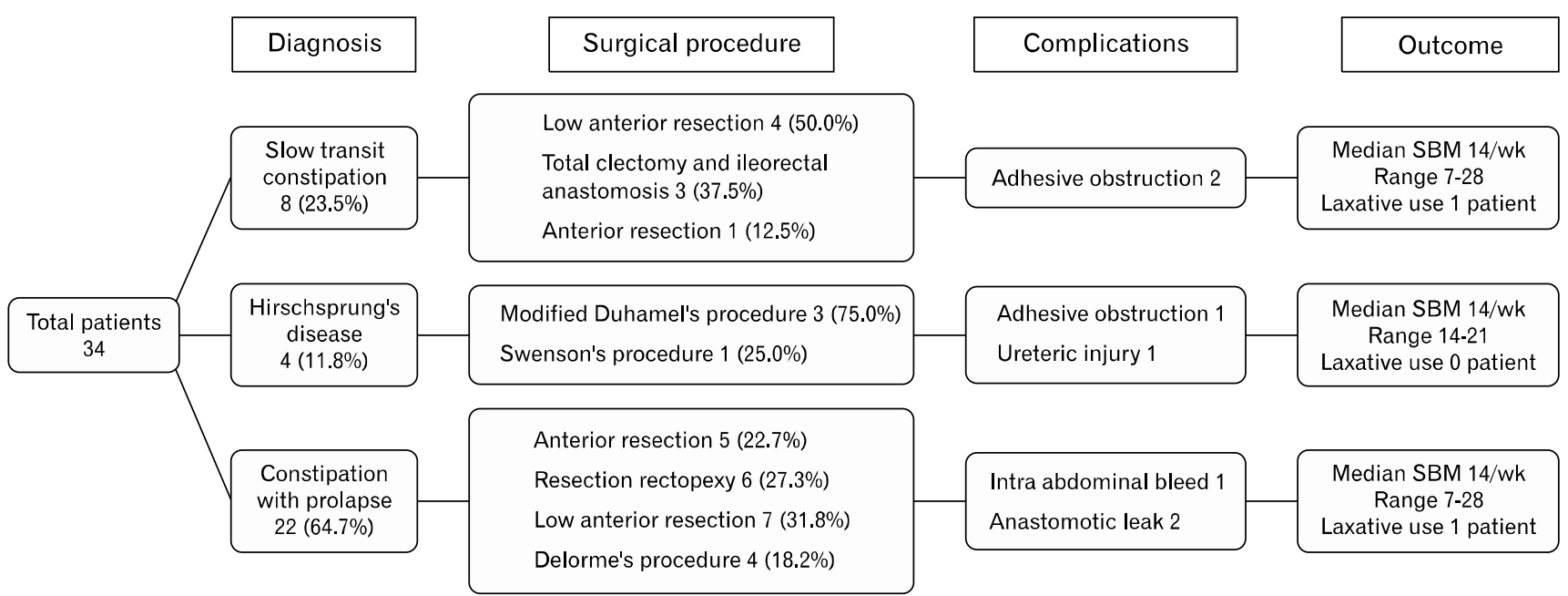

Figure 2. Flow chart showing surgical procedures, complications and early outcome among patients with refractory chronic constipation. SBM, spontaneous bowel movements. 
Table 4. Post Operative Major Complications

\begin{tabular}{ll}
\hline \multicolumn{1}{c}{ Complication } & $\mathrm{n}(\%)$ \\
\hline Anastomotic leak & $2(5.9)$ \\
Intra-abdominal bleed & $1(2.9)$ \\
Adhesive obstruction & $3(8.8)$ \\
Ureteric injury & $1(2.9)$ \\
Total & $7(20.6)$ \\
\hline
\end{tabular}

constipation group) before referral to our center. Two patients with HD had sigmoidectomy; among rectal prolapse group, suture rectopexy and Thiersch wiring were performed on 2 patients, mesh rectopexy in 2, Fothergill's surgery for uterine prolapse in 1 and Thiersch wiring in 1 patient.

After detailed evaluation as described above, patients were taken up for surgical management (Fig. 2). Three patients with STC underwent total colectomy and ileo-rectal anastomosis (IRA), 4 patients underwent low anterior resection (LAR) and 1 underwent anterior resection in view of redundant sigmoid colon on barium enema study. Three out of 4 patients with HD underwent modified Duhamel's procedure while 1 had Swenson's procedure. In patients with constipation and rectal prolapse, anterior resection was done in 5 patients, resection rectopexy in 6, LAR in 7 and Delorme's procedure in 4 patients.

Seven patients $(20.6 \%)$ had major post operative complications (Table 4). However, there was no mortality. One patient had ureter injury in deep pelvis during modified Duhamel's procedure; requiring re-exploration and ureter re-implantation, and recovered well after the surgery. One patient had intra-abdominal bleed which manifested as drain bleed and underwent re-exploration. Three patients had adhesive small bowel obstructions, 2 of them were managed conservatively and one patient required re-exploration. Another 2 patients required re-exploration for anastomotic leak. Six patients had minor complications like wound infection ( 3 patients), post operative fever ( 1 patient) and paralytic ileus ( 2 patients). Out of 8 patients, who were operated elsewhere before referral to our center, 4 patients had complications, of which 3 had major complications; 2 had adhesive obstruction and 1 had ureteric injury.

Follow-up information was available in 27 (79.4\%) patients, and length of follow-up duration varied from 3 to 60 months (mean, 17.8 months). Median SBM significantly improved from 1 per week (range, 0-3 per week) to 14 per week (range, 7-28 per week) following surgical treatment $(P<0.00001)$ (Fig. 2 and 3 ). Two $(5.9 \%)$ patients required laxatives to facilitate the passage of stools intermittently. Overall $25(92.6 \%)$ patients were satisfied

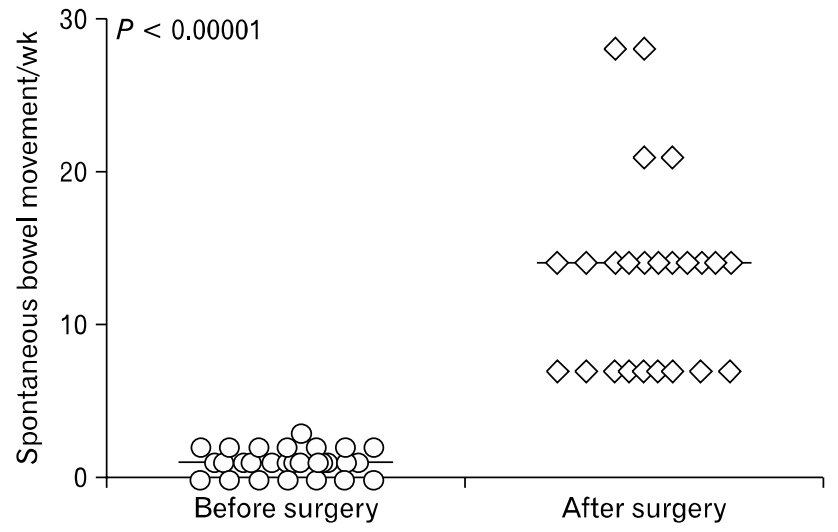

Figure 3. Spontaneous bowel movements before and after surgical treatment (Wilcoxon signed-rank test).

with outcome of surgery. One patient with STC had recurrence of constipation after anterior resection. On subsequent evaluation by CTT study, the patient was diagnosed with colonic inertia and completion total colectomy and IRA was done later. This patient had average of 2 SBMs per day and remained asymptomatic for 2 years of follow-up.

\section{Discussion}

Constipation is a common problem all over the world. The first important step in management of constipation is to exclude organic and anatomic causes with detailed evaluation. Medical management along with biofeedback program is effective in majority of patients with constipation. ${ }^{5}$ Surgery is required in small proportion of patients for correction of anatomical problems like stenotic diverticulitis or outlets problems or specific disorders like $\mathrm{HD}$ or functional disorders like slow transit constipation.

Planning prospective studies related to surgery in refractory constipation is difficult due to the small number of patients. $\mathrm{Pa}$ tients with $\mathrm{CC}$ require thorough medical history, physical and laboratory examination, colonoscopy, barium enema and defecography to rule out the secondary causes of constipation. Barium defecography and anorectal manometry studies are required to evaluate evacuation disorders. Colonic transit studies are necessary to rule out slow transit constipation. When surgical treatment is performed according to results of all the above mentioned investigations, successful outcomes might be achieved. ${ }^{6}$

Different types of surgical procedures have been described in literature, such as total colectomy and IRA (72\%) the most common type, sigmoid colectomy (10\%), subtotal colectomy with ceco-rectal anastomosis $(6 \%)$ and left hemicolectomy $(6 \%){ }^{7}$ In our 
study, total colectomy with IRA and LAR were performed in 4 patients with STC. Laparoscopic total colectomy and IRA have been reported with post operative ileus $(11.4 \%)$, intestinal obstruction $(4.5 \%)$ and satisfaction rate of $88.6 \%$ in post operative period. ${ }^{8}$

Most common early complications following surgery are post operative ileus, infection and anastomotic leak. ${ }^{7}$ Reported incidence of post operative ileus varies from 0 to $13 \%$; infection rate varies from 0 to $11.5 \%$ and anastomotic leak from 0 to $2.3 \%$. $^{8-12}$ In our study, the incidence of post operative obstruction (8.8\%) and anastomotic leak (5.9\%) was similar to reported series. Overall complication rate was $20 \%$.

Knowles et $\mathrm{al}^{13}$ systematically reviewed the literature on colectomy for slow transit constipation in respect to results and outcomes in 1999, which included 32 case series ranging from 12 patients to 106 patients. The commonest postoperative morbidity was small bowel obstruction occurring in 2 to $71 \%$ patients (median, 18\%) which resulted in reoperation in 0 to $50 \%$ (median, $14 \%)$. Similar findings have been observed in our study also with $3(8.7 \%)$ patients having small bowel obstruction among which 1 patient $(2.9 \%)$ required re-exploration. Mortality rates varied from 0 to $6 \%$. None of our patient died in post operative period. Overall documented patient satisfaction rates varied from 39 to $100 \%$ (median, 86\%). Postoperative bowel habit was only numerically quantified in 20 series, with median bowel habits of 2.9/day. Recurrence of constipation symptoms was documented in 0 to $33 \%$, with median of $9 \%$.

In our study, we achieved an excellent satisfaction rate of $92 \%$ with median spontaneous bowel movements of 14 stools per week (range, 7-28 stools per week). Overall reported satisfaction rate in literature was $88 \%$ (57-100\%). Only few studies included objective questionnaires to record improvement and quality of life. Mean stool frequency in the published reports was 19.5 stools per week (range, 7-56 stools per week). ${ }^{7}$

Post operative fecal incontinence as late complication has been reported with incidence of $18 \%$ in literature ranging from 1.3 to $53.0 \%$. $^{7}$ None of our patients experienced incontinence in post operative period. Post operative recurrent constipation was reported in $12.5 \%$ patients $(2-51 \%)$. Requirement of laxatives to relieve constipation has been reported from 0 to $37.5 \%$. $^{7}$ Only 3 $(8.7 \%)$ of our patient had recurrence of constipation, with 2 (5.9\%) requiring laxatives to relieve the symptoms and $1(2.9 \%)$ completion total colectomy.

Long-term functional and quality of life have been evaluated in 104 patients with STC who underwent total colectomy and IRA, with median follow-up period of 11 years. Improvement in constipation in $98 \%$, satisfactory bowel movements in $85 \%$, and improvement in performance measures including social activity $(75 \%)$ and sexual life $(81 \%)$ have been reported. ${ }^{14}$ Same study also reported the improvement in physical and mental health after surgery comparable to normal population. Surgery resulted in durable symptomatic relief and also improvement in long-term quality of life comparable to general population. Cochrane review was done with 12 randomised controlled trials, including 380 patients with rectal prolase, to determine the effects of surgery for the treatment of rectal prolapse in adults. Only two trials with a total of 48 patients addressed the effect of resection on recurrence of rectal prolapse and constipation. The combined results of the study suggested that resection improved constipation and none of the patients had recurrence. ${ }^{15}$

Limitations of our study include selection bias and lack of a control group. Other limitation of the study is the small number of patients. Despite these limitations, our study addresses the issue which is infrequently addressed in Asian patients.

In conclusion, careful preoperative work-up and selection of patients is critical for obtaining good functional results. Surgery should be considered only in patients who have severe refractory constipation after medical treatment. Surgery depends on etiology of constipation. SBM significantly increases following surgical operation, nature of which is dependent on underlying etiology of the constipation and the expertise available. Although the number of study population was small, our study showed meaningful results that surgical management is effective and satisfactory with acceptable morbidity in patients with refractory constipation.

\section{References}

1. Borum ML. Constipation: evaluation and management. Prim Care 2001;28:577-590. vi.

2. Lane WA. Remarks on the result of the operative treatment of chronic constipation. Br Med J 1908;1:126-130.

3. Wald A. Pathophysiology, diagnosis and current management of chronic constipation. Nat Clin Pract Gastroenterol Hepatol 2006;3: 90-100.

4. Ghoshal UC, Gupta D, Kumar A, Misra A. The Colonic transit study by radio-opaque markers to investigate constipation: validation of a new protocol for a population with rapid gut transit. Nat Med J India 2007;20:225-229.

5. Heymen S, Scarlett Y, Jones K, Ringel Y, Drossman D, Whitehead WE. Randomized, controlled trial shows biofeedback to be superior to alternative treatments for patients with pelvic floor dyssynergiatype constipation. Dis Colon Rectum 2007;50:428-441.

6. FitzHarris GP, Garcia-Aguilar J, Parker SC, et al. Quality of life af- 
ter subtotal colectomy for slow transit constipation. Dis Colon Rectum 2003;46:433-440.

7. Arebi N, Kalli T, Howson W, Clark S, Norton C. Systematic review of abdominal surgery for chronic idiopathic constipation. Colorectal Dis 2011;13:1335-1343.

8. Hsiao KC, Jao SW, Wu CC, Lee TY, Lai HJ, Kang JC. Hand-assisted laparoscopic total colectomy for slow transit constipation. Int J Colorectal Dis 2008;23:419-424.

9. Feng $\mathrm{Y}$, Jianjiang L. Functional outcomes of two types of subtotal colectomy for slow-transit constipation: ileo-sigmoidal anastomosis and ceco-rectal anastomosis. Am J Surg 2008;195:73-77.

10. Lahr SJ, Lahr CJ, Srinivasan A, Clerico ET, Limehouse VM, Serbezov IK. Operative management of severe constipation. Am Surg 1999;65:1117-1121, discussion 1122-1123.

11. Platell C, Scache D, Mumme G, Stitz R. A long-term follow-up of patients undergoing colectomy for chronic idiopathic constipation. Aust N Z J Surg 1996;66:525-529.

12. Pemberton JH, Rath DM, Ilstrup DM. Evaluation and surgical treatment of severe chronic constipation. Ann Surg 1991;214:403411.

13. Knowles CH, Scott M, Lunniss PJ. Outcome of colectomy for slow transit constipation. Ann Surg 1999;230:627-638.

14. Hassan I, Pemberton JH, Young-Fadok TM, et al. Ileo-rectal anastomosis for slow transit constipation: long-term functional and quality of life results. J Gastrointest Surg 2006;10:1330-1336, discussion 1336-1337.

15. Tou S, Brown SR, Malik AI, Nelson RL. Surgery for complete rectal prolapse in adults. Cochrane Database Syst Rev 2008;(4): CD001758. 